\title{
Dwell Time Algorithm Based on Bounded Constrained Least Squares Under Dynamic Performance Constraints of Machine Tool in Deterministic Optical Finishing
}

\author{
Yunfei Zhang ${ }^{1,2} \cdot$ Fengzhou Fang $^{2} \cdot$ Wen Huang $^{1} \cdot$ Wei Fan $^{1}$
}

Received: 4 February 2020 / Revised: 7 December 2020 / Accepted: 17 December 2020 / Published online: 13 January 2021

(c) The Author(s) 2021

\begin{abstract}
The dwell time algorithm is one of the most important techniques within the deterministic optical surfacing technologies. The existing dwell time algorithms are generally based on non-negative least squares (NNLS) without considering the dynamic performance constraints of machine tools. This is a circumstance that leads to poor convergence accuracy. In this paper, a dwell time algorithm, based on bounded constrained least-squares (BCLS) under dynamic performance constraints of the machine tool, has been developed. The upper and lower constraints of the dwell time model could be derived through the acceleration and deceleration mechanism of the CNC (Computer Numerical Control) machine tools. A two-metric projection Newton iteration algorithm was used to solve the large-scale dwell time model, which greatly improved the computation efficiency. The results of the experiments and simulations showed that the proposed algorithm will give a very high convergence accuracy for optical finishing with machine tools with different dynamic performances. When the machine acceleration was set to a value as low as $0.1 \mathrm{~g}$, the accuracies of the surface figures PV (Peak-to-Valley) and RMS (Root Mean Square) till improved by $40.8 \%$ and $55.2 \%$, respectively, when using the BCLS algorithm. The influences of different dynamic performances of the machine tools on the dwell time solutions have also been investigated, which will provide a good guidance in the design of deterministic polishing machine tools.
\end{abstract}

Keywords Magnetorheological finishing $\cdot$ Deterministic polishing technologies $\cdot$ Dwell time algorithm $\cdot$ Bounded constrained total least squares

\section{Introduction}

With the rapid increase of requirements for the fabrication of high-precision optical elements in advanced optical systems, several advanced deterministic optical finishing technologies have been developed over the past decades (e.g., computer controlled optical surface finishing (CCOS) [1], magnetorheological finishing (MRF) [2, 3], ion beam figuring (IBF) $[4,5]$, bonnet polishing (BP) $[6,7]$ and other polishing technologies [8-11]). These technologies make use of the

Fengzhou Fang

fzfang@tju.edu.cn

1 Institute of Machinery Manufacturing Technology, China Academy of Engineering Physics, Mianyang 621900, China

2 State Key Laboratory of Precision Measuring Technology \& Instruments, Centre of Micro/Nano Manufacturing Technology- MNMT, Tianjin University, Tianjin 300072, China controllable removal characteristics of polishing tools, to accurately control the dwell time distribution and thereby ensuring a high convergence accuracy of the full-range band surface error of the optics.

In order to obtain nano-level polishing accuracy, machine tool designers always try to improve the machine tool accuracy of deterministic polishing equipment as high as possible. However, too high machine tool accuracy will not only greatly increase the machine tool cost, but may not be of much significance to improve the polishing accuracy. It is mainly because, for ultra-precision polishing equipment, the polishing accuracy is not directly related to the motion accuracy of the machine tool, but the control performance of dwell time is the most important factor. Thus, the dwell time algorithm is one of the most important techniques in modern optical manufacturing technologies $[12,13]$.

In deterministic optical finishing technologies, the amount of material removal can be expressed as discrete two-dimensional convolution operations of the dwell time 
function and the removal function [14]. The dwell time algorithm is used to solve the deconvolution process and to ensure that the calculated dwell time not only meets the performance of machine tools, but also has high surface error convergence efficiency. Various dwell time algorithms have earlier been proposed, including the Fourier transform method [15], the numerical iteration method [16-18], the matrix equation method $[19,20]$, and other algorithms [21, 22]. The dwell time algorithm being based on the matrix equation is the most widely adopted one. It transforms the two-dimensional deconvolution model of the dwell time into a simple form of a matrix equation, which is more flexible than the discrete deconvolution model. In addition, it is not limited by the polishing spot shape and polishing path [23].

A variety of matrix equation-based dwell time algorithm models have been proposed, to continuously improve the comprehensive performances and to promote applications of the dwell time algorithm. Carnal et al. firstly established a dwell time model based on the matrix equation, which utilized a least square $\mathrm{QR}$ decomposition (LSQR) method in obtaining a theoretical solution [19]. However, the solution could not satisfy the non-negative constraints and needed further post-processing, which would reduce the convergence efficiency. Lee et al. described a dwell time algorithm which was based on Non-Negative Least-Squares (NNLS) for the polishing of small-sized axis-symmetrical aspheric surfaces. The non-negative constraint conditions of the dwell time was then considered to minimize the residual surface errors [24]. The non-negative constrained optimization algorithm has a remarkably high theoretical convergence accuracy of surface errors and is a promising algorithm. Meanwhile, the common problems of the matrix equationbased dwell time algorithm have also been recognized (e.g., ill-conditioned characteristics, low computational efficiency and low convergence accuracy in engineering, and restrictions in the polishing of large-aperture or irregularly shaped workpieces).

By focusing on the control of the ill-conditioned matrix equation, Wu et al. [23], Deng et al. [25], and Dong et al. [26] did independently explore the Tikhonov regularization method with the purpose to control the ill-condition of the matrix equation. Different damped factor optimization methods were then given. Zhou et al. verified that optimal removal can be determined at the corner of the curve in the figure showing the process times vs. residual figure errors (of type RMS) [27], which gives an excellent performance in balancing the process time with the residual figure error. By focusing on an improved computational efficiency, Li et al. proposed an algorithm for the solution of a large scale and sparse matrix equation, which was based on the Subsapzilai and Borwein method for processing large aperture optical elements [28]. In addition, Dong et al. proposed a constrained LSQR method (based on the sparse matrix) to largely reduce the memory cost and to improve the computational efficiency [29]. By focusing on an improved convergence accuracy, Song et al. established a constrained non-linear optimization model by treating the limitations of the dynamic performances of the machine as constraint conditions [30]. Both the 2-norm of the residual surface error, and the dwell time gradient, were then taken into consideration as objective functions. This algorithm has theoretically resulted in a much high convergence accuracy and adaptability to machine tools. Nevertheless, the non-linear version of the optimization algorithm is far too complex. Not only is the dynamic performance of the machine difficult to model, but the computational efficiency of the non-linear optimization model can also be low (especially for large-aperture optical elements).

After more than a decade of development, the matrix equation-based dwell time algorithm, with non-negative constraints, has been proven to be efficient in reducing illconditioning and to improve the computational efficiency. However, the algorithm does not consider the dynamic performances of machine tools, and this doesn't make the convergence accuracy large enough. It also limits the universality. Thus, designers must continue working with the improvement of the accuracy of the equipment.

To improve the polishing accuracy of the surface error, and the adaptability to any machine tool, a Bounded Constrained Least-Square (BCLS)-based dwell time algorithm is here being proposed. The dynamic performance constraints of machine tools are thereby considered. The upper and lower constraints of the dwell time model have then, through the acceleration and deceleration mechanisms, been derived. Thus, the dwell time solution will automatically satisfy the special requirements by different machine tools. In addition, the Tikhonov regularization has also been applied to solve the ill-posed problem [31, 32]. Also, the regularization parameter has, without any prior knowledge, been determined by using an adaptive method. The numerically calculation method of the BCLS-based dwell time model makes use of the sparse characteristics of the removal factor matrix. It greatly improves the computational efficiency, as well as reduces the storage cost. Simulations and experimental verifications confirm that the algorithm has an exceedingly high convergence accuracy, to be used for optical finishing machine tools with different dynamic performances.

\section{Dwell Time Model}

\subsection{BCLS-Based Dwell Time Algorithm Model}

In the process of deterministic polishing for optical elements, the polishing tool does continuously scan the workpiece surface along a certain polishing path (e.g., raster, 
spiral, etc.) [33]. This is demonstrated in Fig. 1). The total amount of removed material in any given surface area can be obtained as the summary of removed material from polishing at all path points.

The amount of material removed at every control point is shown as follows [30]:

$h\left(x_{k}, y_{k}\right)=\sum_{i=1}^{N_{t}} r\left(x_{k}-x_{i}, y_{k}-y_{i}\right) t\left(x_{i}, y_{i}\right) \quad k=1, \ldots, N_{h}$

where $t\left(x_{i}, y_{i}\right)$ denotes the dwell time at the $i$ th path point, $h\left(x_{k}, y_{k}\right)$ is the desired amount of removed material at the $k$ th figure-control point, $N_{t}$ is the total number of the path points, $N_{h}$ is the total number of the figure-control points, and $r\left(x_{k}-x_{i}, y_{k}-y_{i}\right)$ is the amount of removed material at the $k$ th figure-control point (when the center of the polishing tool dwells at the $i$ th path point).

From here on, $t\left(x_{i}, y_{i}\right), h\left(x_{k}, y_{k}\right)$, and $r\left(x_{k}-\xi_{i}, y_{k}-\eta_{i}\right)$ will be denoted as $t_{i}, h_{k}, r_{k i}$ respectively. Moreover, the dwell time vector, material removal vector, and removal factor matrix
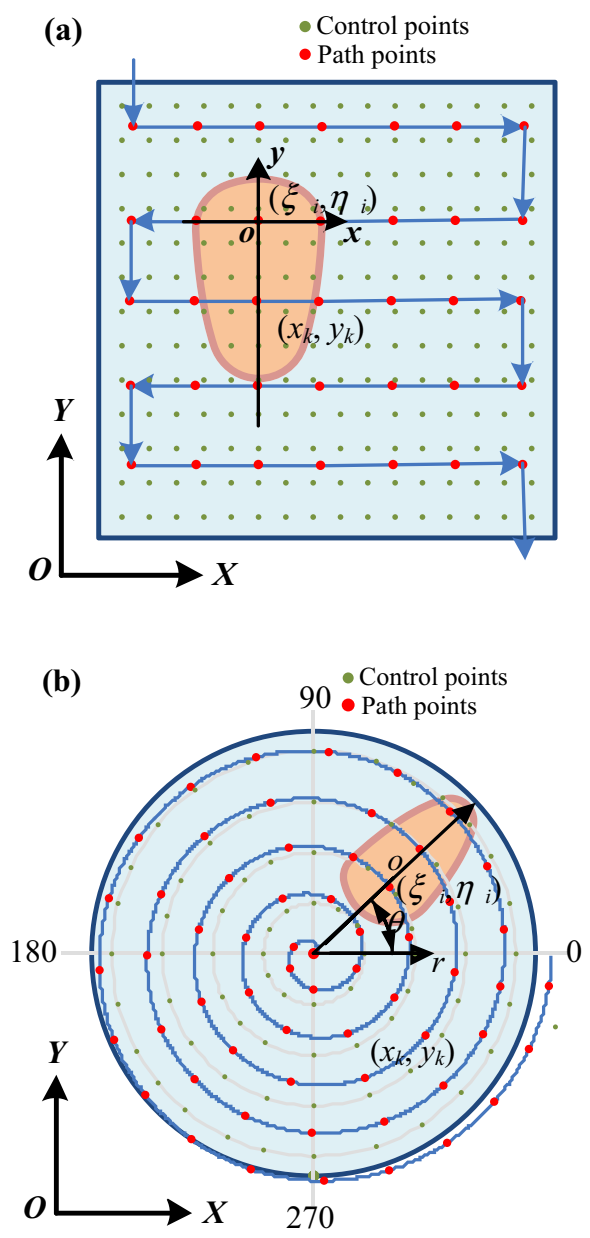

Fig. 1 Diagram of polishing path and figure-control point distribution. a Raster path, and (b) spiral path
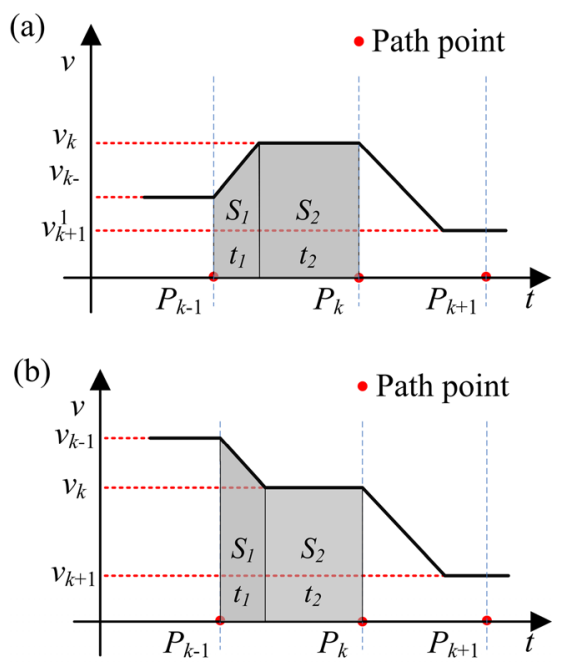

Fig. 2 Speed interpolation algorithms at different stages. a Speed increase stage, and (b) speed reduction stage

will be denoted as $\boldsymbol{t}, h$, and $\boldsymbol{R}$, respectively. Equation (1) can thereby be obtained as [31]:

$R t=h$

In Eq. (2),

$t=\left[\begin{array}{c}t_{1} \\ t_{2} \\ \vdots \\ t_{N_{t}}\end{array}\right], h=\left[\begin{array}{c}h_{1} \\ h_{2} \\ \vdots \\ h_{N_{h}}\end{array}\right], R=\left[\begin{array}{cccc}r_{11} & r_{12} & \cdots & r_{1 N_{t}} \\ r_{21} & r_{22} & \cdots & r_{2 N_{t}} \\ \vdots & \vdots & \vdots & \vdots \\ r_{N_{h} 1} & r_{N_{h} 2} & \cdots & r_{N_{h} N_{t}}\end{array}\right]$

The desired material removal vector, $\boldsymbol{h}_{\mathbf{0}}$, at the figurecontrol points can be obtained through the initial surface error of the workpiece. Based on the polishing spot and path, the removal factor matrix, $\boldsymbol{R}$, can also be calculated. Let us now assume that $\boldsymbol{h}=\boldsymbol{h}_{\mathbf{0}}$, and that the dwell time function can be deduced by solving Eq. (2).

In general, Eq. (2) is an overdetermined equation with no exact solution. The dwell time is realized by controlling the speed of the movable axis, which is limited by the acceleration of the machine tool. When the calculated dwell time does not match the acceleration and speed of the machine tool, the convergence accuracy will decrease, or even deteriorate. Thus, the dwell time needs to meet the dynamic constraint conditions of the machine tool [34].

The existing dwell time algorithms only consider the nonnegative constraints of the dwell time and ignore the upper and lower bound constraints of the speed and accelerations of the machine tool. To improve the convergence efficiency of the dwell time algorithm and resolve the matching problems between the algorithm and the dynamic performance of the machine tool, a BCLS-based dwell time model is here proposed: 


$$
\begin{array}{ll}
\min & \frac{1}{2}\left\|\mathbf{R t}-\mathbf{h}_{\mathbf{0}}\right\|_{2}^{2}+\frac{1}{2} \beta\|\mathbf{t}\|_{2}^{2} \quad(\beta>0) \\
\text { s.t. } & \mathbf{t}_{\mathbf{l}} \leq \mathbf{t} \leq \mathbf{t}_{\mathbf{p}}
\end{array}
$$

In Eq. (4), $\beta$ is the Tikhonov regularization factor, and $\mathbf{t}_{\mathbf{1}}$ and $\mathbf{t}_{\mathbf{p}}$ are the upper and lower limit constraints of the solutions of the dwell time, $\mathbf{t}$, respectively. These limits are based on the dynamic limitations of the machine. Moreover, $\|\bullet\|_{2}$ is the definition of the 2-norm of a vector.

The model described by Eq. (4) considers the sparse characteristics of the removal factor matrix, with the purpose to improve the efficiency of the computations. It also introduces a damping factor to reduce ill-conditioning and applies bound constraints on the dynamic performance constraints of the machine tools. Thus, this model has a much high accuracy and accessibility for computational solutions.

\subsection{Dynamic Performance Constraints of Machine Tools}

When the movable axis moves from one point to another along the path, it will, at first, be accelerated (or decelerated) and then reach a steady speed. Speed interpolation algorithms may be different for different CNC systems. However, the speed interpolation process can be simplified to become linear for the acceleration stage [35] (as illustrated in Fig. 2).

When the machine tool moves from path point $\mathrm{P}_{k-1}$ to $\mathrm{P}_{k}$, the motion-related velocities and durations can be written:

$\left\{\begin{array}{l}\left(v_{k}+v_{k-1}\right) t_{1} / 2+v_{k} t_{2}=S_{k} \\ v_{k}-v_{k-1}=\mathbf{a} t_{1} \\ t_{1}+t_{2}=t_{k}\end{array}\right.$

Here, $t_{1}$ is the acceleration duration (or deceleration), $t_{2}$ is the duration in the constant speed segment, and $t_{k}$ is the dwell time. Moreover, $v_{k-1}$ and $v_{k}$ are the previous and current speeds at the path point, $S_{k}$ is the distance between $\mathrm{P}_{k-1}$ and $\mathrm{P}_{k}$, and $\mathbf{a}$ is the acceleration of the machine tool. Let us now assume that $a=|\mathbf{a}|$. It then follows that $\mathbf{a}=\mathbf{a}$ in the acceleration stage, and that $\mathbf{a}=\mathbf{- \mathbf { a }}$ in the deceleration stage.

In solving Eq. (5), the result will be given as follows:

$$
\left\{\begin{array}{l}
t_{1}=t_{k}-\sqrt{t_{k}^{2}-\frac{2\left(S_{k}-v_{k-1} t_{k}\right)}{\mathbf{a}}} \\
t_{2}=\sqrt{t_{k}^{2}-\frac{2\left(S_{k}-v_{k-1} t_{k}\right)}{\mathbf{a}}} \\
v_{k}=v_{k-1}+\mathbf{a}\left[t_{k}-\sqrt{t_{k}^{2}-\frac{2\left(S_{k}-v_{k-1} t_{k}\right)}{\mathbf{a}}}\right]
\end{array}\right.
$$

Since $v_{k}$ needs to meet the constraint conditions at the upper and lower speed limits, it can be written as: $v_{\text {min }} \leq v_{k-1}+\mathbf{a}\left[t_{k}-\sqrt{t_{k}^{2}-\frac{2\left(S_{k}-v_{k-1} t_{k}\right)}{\mathbf{a}}}\right] \leq v_{\max }$

Since the speed at the previous path point is unknown prior to the dwell time calculations, Eq. (7) cannot be directly used to obtain the dwell time. At the acceleration stage, to ensure that the machine tool will have enough time to accelerate, the sufficient condition should be:

$\frac{1}{2}\left(v_{k-1}+v_{k}\right) t_{k}=\frac{1}{2} a t_{k}^{2}+v_{k-1} t_{k} \geq S_{k}$

In this case, $\boldsymbol{a}=\mathrm{a}$. In solving Eq. (8), the calculated dwell time, $t_{k}$, should meet the following condition:

$t_{k} \geq\left(-v_{k-1}+\sqrt{v_{k-1}^{2}+2 a S_{k}}\right) / a$

Assuming that Eq. (9) is valid when $v_{k-1}=v_{\text {min }}$, then it will also be valid for any $v_{k-1}$. This gives:

$t_{k} \geq\left(-v_{\min }+\sqrt{v_{\min }^{2}+2 a S_{k}}\right) / a$

We have thereafter to ensure that the speed, $v_{k}$, satisfies Eq. (7) even after the acceleration stage. Within this stage, if Eq. (7) is valid for $v_{k-1}=v_{\min }$, then it should be valid for any other value of $v_{k-1}$. The machine tool should otherwise decelerate. Therefore, the sufficient conditions for Eq. (7) are as follows:

$0 \leq t_{k}-\sqrt{t_{k}^{2}-\frac{2\left(S_{k}-v_{\min } t_{k}\right)}{a}} \leq \frac{v_{\max }-v_{\min }}{a}$

Here, the constraints of the dwell time, $t_{k}$, can be simplified as:

$\frac{S_{k}}{v_{\max }}+\frac{\left(v_{\max }-v_{\min }\right)^{2}}{2 v_{\max } a} \leq t_{k} \leq \frac{S_{k}}{v_{\min }}$

In the deceleration stage, to ensure that the machine tool has enough time to decelerate, the sufficient condition should be satisfied as follows:

$\frac{1}{2}\left(v_{k-1}+v_{k}\right) t_{k}=-\frac{1}{2} a t_{k}^{2}+v_{k-1} t_{k} \leq S_{k}$

In this case, $\mathbf{a}=-a$. In solving Eq. (13), the calculated dwell time, $t_{k}$, should meet the following condition:

$t_{k} \leq\left(v_{k-1}-\sqrt{v_{k-1}^{2}-2 a S_{k}}\right) / a$

Let us now assume that Eq. (14) is valid when $v_{k-1}=v_{\text {min }}$, then Eq. (14) will always be valid for any $v_{k-1}$. This gives: 
$t_{k} \leq\left(v_{\max }-\sqrt{v_{\max }^{2}-2 a S_{k}}\right) / a$

It is now important to ensure that the speed, $v_{k}$ satisfies Eq. (7) after deceleration. In the deceleration stage, if Eq. (7) is valid when $v_{k-1}=v_{\max }$, then Eq. (7) should always be valid for any value of $v_{k-1}$. The machine tool should otherwise accelerate. Therefore, the sufficient conditions for Eq. (7) can be simplified as:

$\frac{S_{k}}{v_{\max }} \leq t_{k} \leq \frac{S_{k}}{v_{\min }}-\frac{\left(v_{\max }-v_{\min }\right)^{2}}{2 v_{\min } a}$

However, it is not known whether the current path points are in the acceleration, or deceleration, stage in the process of dwell time calculation. To ensure that the dwell time meets all necessary requirements, the dwell time constraint conditions can be uniformly expressed as:

$\frac{S_{k}}{v_{\max }}+\frac{\left(v_{\max }-v_{\min }\right)^{2}}{2 v_{\max } a} \leq t_{k} \leq \frac{S_{k}}{v_{\min }}-\frac{\left(v_{\max }-v_{\min }\right)^{2}}{2 v_{\min } a}$

In Eq. (17), the upper and lower limits of dwell time should satisfy the relative relationship as follows:

$\frac{S_{k}}{v_{\max }}+\frac{\left(v_{\max }-v_{\min }\right)^{2}}{2 v_{\max } a} \leq \frac{S_{k}}{v_{\min }}-\frac{\left(v_{\max }-v_{\min }\right)^{2}}{2 v_{\min } a}$

Eq. (18) can thereafter be simplified to:

$v_{\max }^{2}-v_{\min }^{2} \leq 2 S_{k} a$

Moreover, the characteristic speed can be defined as:

$v^{*}=\sqrt{\frac{2 S_{k} a}{1-f^{2}}}$

where $f$ is the modulation ratio of the minimum-to-maximum speed, and $v_{\min }=f \cdot v_{\max }$. The maximum speed cannot be larger than the characteristic speed to ensure the validity of the dwell time constraint conditions.

Eq. (17) is thereafter substituted into the dwell time model described by Eq.(4), and, thus, a complete dwell time mathematical model has been established.

\section{Numerical Calculation Method}

\subsection{Algorithm Implementation}

In addition to the proposed dwell time model, a dwell time algorithm has also been developed in the present study. A sketch over the corresponding flow chart is shown in Fig. 3. At first, paths have been planned with discretized control

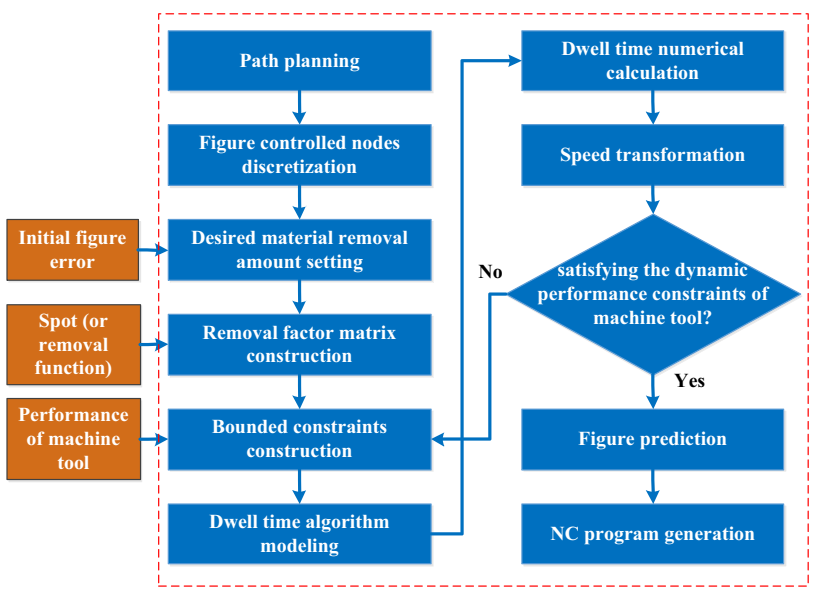

Fig. 3 Flow chart of the dwell time algorithm

points. The coordinates of these path points, and of surface error-control points, have thereafter been determined. Secondly, the dwell time optimization model (based on BCLS) was established based on the initial surface error of the workpiece, the polishing spot, and the dynamic constraint performance parameters of the machine tool. Thirdly, a BCLS solver was included for the implementation of the model, with the presentation of the resulting dwell time distribution. During the calculation process, the dwell time was automatically converted into the speed of the axis in the machine. This was made to judge whether the speed meets the dynamic performance constraints of the machine tool, or not. If this is not the case, the boundary conditions should be modified with a following re-calculation of the dwell time. If successful, a surface figure prediction should be done with a following generation of an NC program for machining.

\subsection{BCLS Solver}

The model proposed in Eq. (4) involves a least-square optimization problem with bound constraints. To facilitate the numerical solving of this model, a general form of the algorithm could be mathematically described as shown below:

$$
\begin{array}{ll}
\min & \frac{1}{2}\|\mathbf{A x}-\mathbf{b}\|_{2}^{2}+\frac{1}{2} \beta\|\mathbf{x}\|_{2}^{2} \quad(\beta>0) \\
\text { s.t. } & \mathbf{x}_{\mathbf{l}} \leq \mathbf{x} \leq \mathbf{x}_{\mathbf{u}}
\end{array}
$$

where $\mathbf{A}$ is an $m \times n$ matrix that is equivalent to the removal factor matrix, R. Furthermore, $\mathbf{x}$ is an $n \times 1$ vector, being equivalent to the dwell time vector, $\mathbf{t}$. Finally, $\mathbf{b}$ is the $m \times 1$ vector that is equivalent to the target removal vector, $\mathbf{h}$, at the control points.

There are many numerical calculation algorithms by which it is possible to solve the algorithm model described by Eq. (21). However, most of them cannot meet the 
large-scale solving requirements of a sparse matrix, and therefore leads to a low computation efficiency. In the preferred algorithm, a mature two-metric projection Newton iteration algorithm has been used to guarantee both accuracy and efficiency. After that the parameters $\mathbf{A}, \mathbf{b}, \mathbf{x}_{\mathbf{l}}, \mathbf{x}_{\mathbf{u}}, \beta$ have been obtained, a mathematical model for solving the dwell time can be established. The flow chart for the numerical dwell time calculation algorithm is shown in Fig. 4.

The stability of the solution is strongly correlated with $\beta$. However, a larger value of $\beta$ will decrease the convergence accuracy of the solution. Hence, to find the optimal $\beta$ value, the method must use the square of the 2-norm of the residual surface error, Rt- $\mathbf{h}_{\mathbf{0}}$, as an abscissa. In addition, the method must also use the square of the 2-norm of the dwell time, $\mathbf{t}$, as the ordinate, as well as the Tikhonov regularization, $\beta$, as a variable. As a result, the $\beta$ parameter will form a curve, which is named the L-curve [36]. The optimal value of $\beta$ can then be chosen from the most pronounced curvature of the L-curve.

\section{Validation and Discussion}

\subsection{Experiments}

\subsubsection{Validation of the BCLS-Based Algorithm}

To validate the reliability of the BCLS-based dwell time algorithm, some polishing experiments on a $200 \times 200 \mathrm{~mm}^{2}$ fused silica workpiece have been conducted by using the MRF technology. The measured initial surface error of the

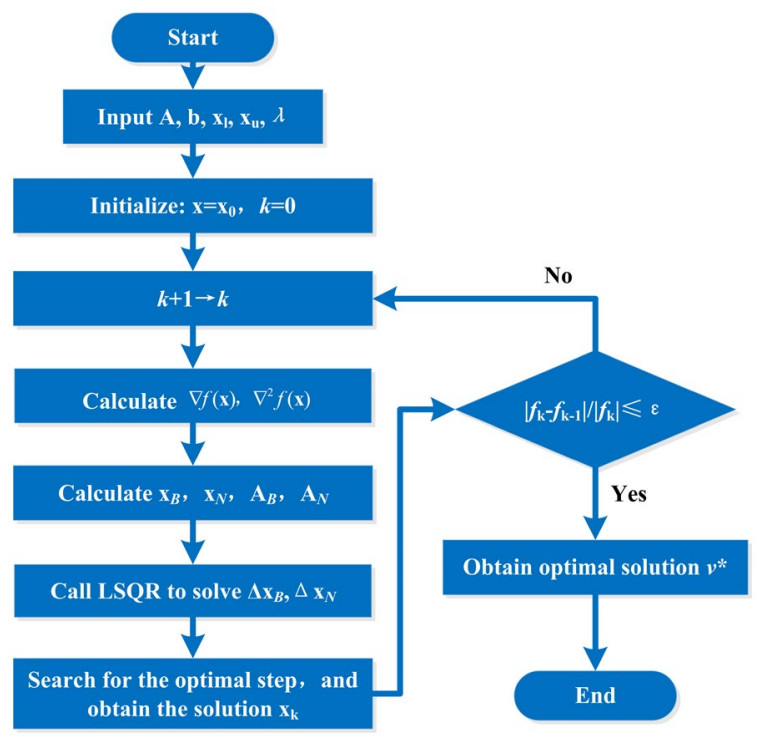

Fig. 4 Flow chart showing the numerical dwell time calculation algorithm workpiece and the MRF spot (i.e., the removal function) are shown in Fig. 5. The initial surface error was $0.452 \lambda$ (of type PV) ( $\lambda$ is a wavelength unit, being equal to approximately $0.6328 \mu \mathrm{m}$ ), and $0.0663 \lambda$ (of type RMS). The spot had a dimension of $13.89 \mathrm{~mm}$ in length, and $6.86 \mathrm{~mm}$ in width. Also, the peak removal rate (PRR) was $6.63 \lambda / \mathrm{min}$, and the volume removal rate (VRR) was $0.193 \mathrm{~mm}^{3} / \mathrm{min}$.

The experiments were carried out on an in housedeveloped MRF machine (as shown in Fig. 6). It has two combined polishing heads and can finish an aspheric lens with an aperture as large as $1.2 \mathrm{~m}$. The maximum allowed acceleration of the main movable axis is $0.3 g(\approx 2.94 \mathrm{~m} /$ $\mathrm{s}^{2}, g$ is the gravitational acceleration), with a speed range of $200-4000 \mathrm{~mm} / \mathrm{min}$.

The raster line was chosen to be a tool path, and the manufactured control points were evenly separated with $1 \mathrm{~mm}$ spacing. The horizontal and longitudinal spaces in the raster line were of size $1.5 \mathrm{~mm}$ and $2.09 \mathrm{~mm}$, respectively. Based on Eq. (17), the constraint condition of any dwell point was within the following range:

$0.04157 \mathrm{~s} \leq t_{k} \leq 0.4222 \mathrm{~s}$

The dwell time calculations were performed by using the self-developed MRF software. The theoretically predicted values of dwell time distribution and speed distribution are shown in Fig. 7. The distribution of the dwell times was (a)

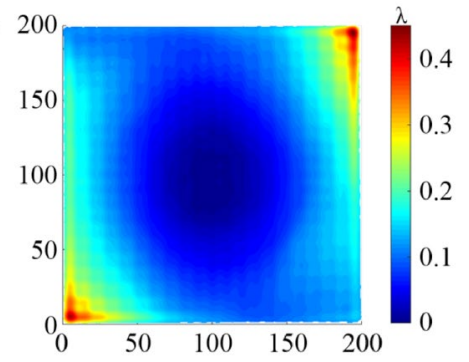

(b)

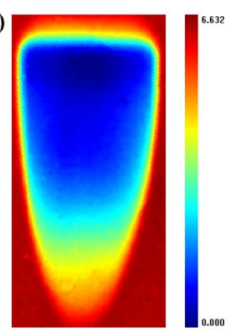

Fig. 5 a Initial surface error of the workpiece. b MRF spot

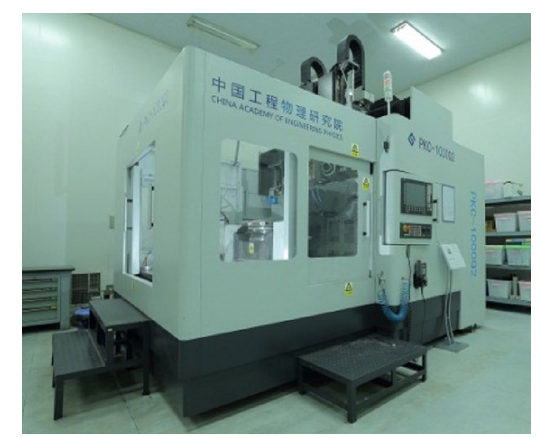

Fig. 6 Self-developed MRF machine 
(a)

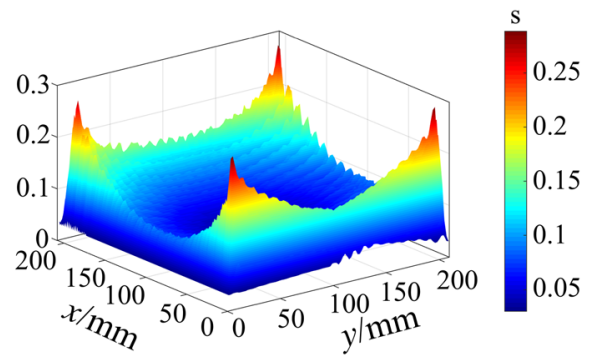

(b)

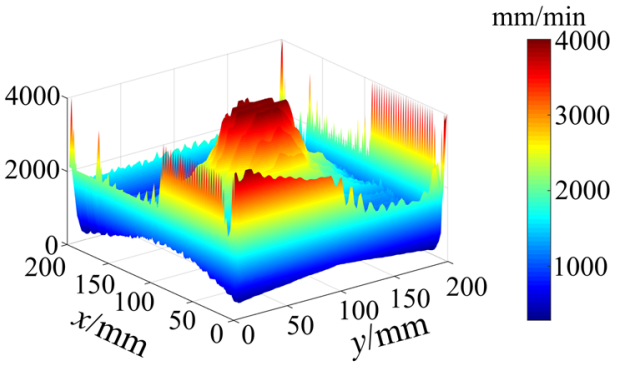

Fig. 7 Results from dwell time and speed distribution calculations. a Dwell time distribution, and (b) speed distribution

observed to coincide with the distribution of the initial surface error (with longer dwell times close to the boundaries and shorter in the central area). On the contrary, the speed distribution was just the opposite with lower speeds closer to the boundaries and higher speeds in the center.

By chosing an arbitrary slice in the respective distribution (in parallell with either the $\mathrm{x}$ - or $\mathrm{y}$-axis), the following pattern can be observed (see Fig. 8). It can be seen that the dwell time distribution is strictly bound by Eq.

(a)

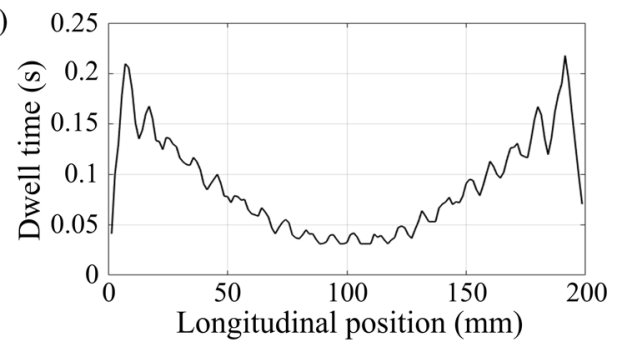

(b)

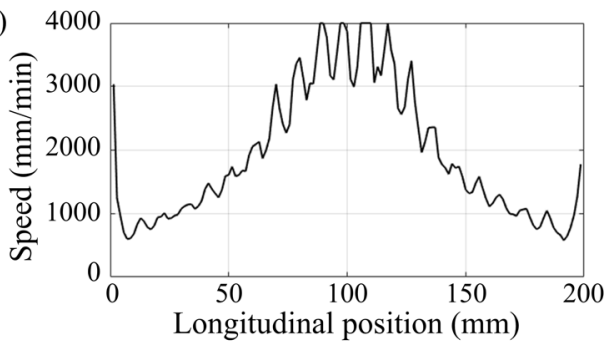

Fig. 8 Calculated results of the (a) dwell time and (b) speed for an arbitrary slice through the $\mathrm{x}$ - or $\mathrm{y}$-axis
(22) (Fig. 8a), and that the calculated speed is located within the range $200-4000 \mathrm{~mm} / \mathrm{min}$ (see Fig. 8 b). The result is consistent with the expectation from the dwell time algorithm.

MRF polishing experiments, on a workpiece, have also been carried out in the present study. The design of these experiments was based on the results from the numerical calculations of the dwell time, which were, furthermore, verified by theoretical simulations. The predicted residual surface error is shown in Fig. 9a, with a flat surface error of $0.0349 \lambda$ (of type PV) and $0.00142 \lambda$ (of type RMS). The duration of the polishing process was approximately $32 \mathrm{~min}$. The residual surface error of the experimental result is shown in Fig. 9b. The surface figure accuracy was improved to $0.099 \lambda$ (of type PV) and $0.012 \lambda$ (of type RMS), with the convergence efficiency remarkably increased by a factor of 4.57 and 5.52, respectively. These results are indications on the feasibility of the proposed dwell time algorithm. However, there still exists a certain difference between the predicted residual surface error and that of the experiment results. This is mainly due to the characterization error and fluctuation of the removal function in the actual polishing process. (a)

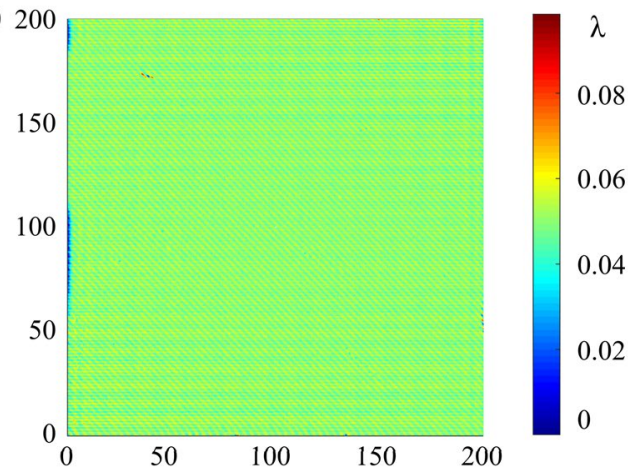

(b)

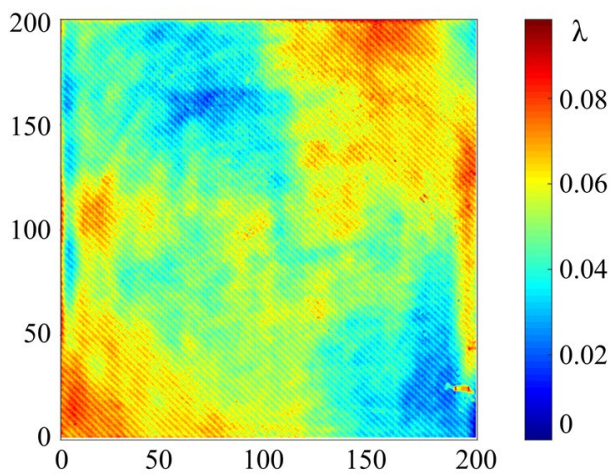

Fig. 9 Residual surface errors of (a) predicted results $(0.034944 \lambda$ of type PV; $0.00142 \lambda$ of type RMS), and (b) experimental results (0.099 $\lambda$ of type PV; $0.01199 \lambda$ of type RMS) 


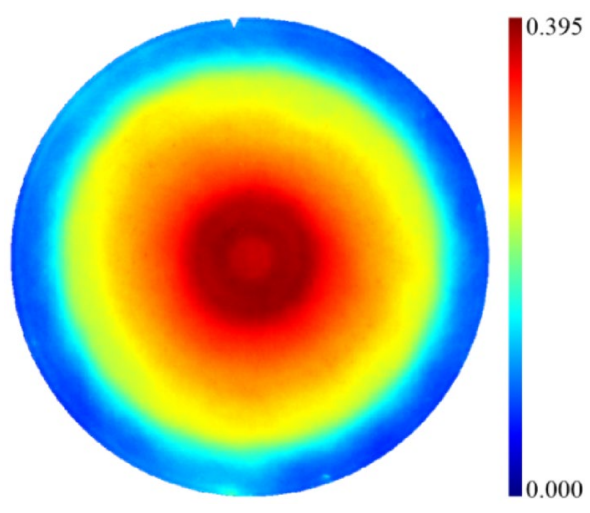

Fig. 10 Initial surface error of the workpiece

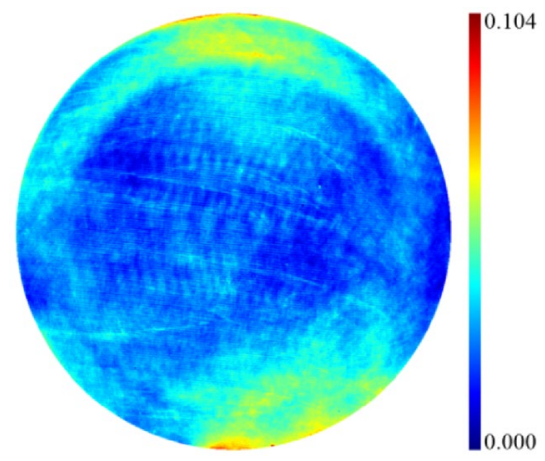

Fig. 11 Experimental results for the $\varphi 285 \mathrm{~mm}$ workpiece

\subsubsection{Validation of the Convergence Accuracy of the BCLS-Based Dwell Time Algorithm}

In order to verify that the proposed algorithm also has a high convergence efficiency for low-precision machine tools, the allowed maximum acceleration of the main movable axis (i.e., of the MRF machine tool) was set to $0.03 \mathrm{~g}$, which is only one-tenth of normal conditions. The other parameters remained unchanged. A $\varphi 285 \mathrm{~mm}$ fused silica plane was selected, with an initial surface figure accuracy of $0.394 \lambda$ (of type PV) and $0.0922 \lambda$ (of type RMS) (as shown in Fig. 10).

The raster line was chosen to be a tool path, and the manufactured control points were evenly distributed with $1 \mathrm{~mm}$ spacing. The horizontal and longitudinal spaces in the raster line were of size $1.5 \mathrm{~mm}$ and $2.4975 \mathrm{~mm}$, respectively. After 40.56 min' polishing, the surface error of the workpiece was improved to $0.104 \lambda$ (of type PV) and 0.0111 $\lambda$ (of type RMS), with the convergence efficiencies remarkably increased by a factor of 3.78 and 8.30 , respectively (as shown in Fig. 11). These experimental results show that the algorithm proposed in the present paper also has high convergence accuracy for machine tools with extremely low dynamic performances.

\subsubsection{Repetitive Performance Verification of the BCLS-Based Dwell Time Algorithm.}

In addition to the development of a dwell time algorithm, the author has also developed an MRF process software. It runs on different types of MRF machine tools and can successfully process several optics with different aperture and shape. The convergence efficiency may, though, be different from the experimental ones. This is most probably, due to different material characteristics, and initial surface errors of workpieces, in practical engineering applications. The convergence efficiency of the dwell time algorithm has been shown to be between 2.5 and 4.8 (by using RMS), with an averaged convergence efficiency of up to 3.3 . Therefore, the convergence performance of the algorithm has been fully verified and has an excellent repeatability.

\subsection{Comparison and Discussion}

Theoretically, the BCLS-based dwell time algorithm automatically satisfies the speed and acceleration constraints without any subsequent post-processing. Thus, it has a higher convergence efficiency and there are no special requirements for the performance of machine tools. To verify the convergence accuracy of the BCLS-algorithm, some comparable simulations have been conducted in analyzing the computational performance of this algorithm, as compared with that of the classical NNLS algorithm. In addition, the influence of different acceleration and speed on the convergence accuracy of the surface error was also investigated. In these simulations, the initial surface error, polishing spot, path parameters, and speed range were all identical to the parameters in the experiment we intended to compare with.

\subsubsection{Convergence Accuracy of the BCLS-Based Dwell Time Algorithm}

To obtain the convergence accuracy of the BCLS-based dwell time algorithm, five dwell time calculations, with different machine acceleration limitations, were selected. These results were compared with corresponding results obtained by using the classical NNLS algorithm. The NNLS algorithm has a post-processing step (i.e., after that the dwell time has been calculated) to guarantee that the speed and acceleration can meet the machine tool constraints. In the simulations, the acceleration of the machine tool was set to increase from $0.05 \mathrm{~g}$ to $0.8 \mathrm{~g}$ ( $\mathrm{g}$ is acceleration constant of gravity $-9.8 \mathrm{~m} / \mathrm{s}^{2}$ ). In addition, the speed constraint condition was set to $0.8 v^{*}$, and the Tikhonov regulation factor was set to 0 . The simulation results, obtained by using different dwell time algorithms, is shown in Table 1.

As can be seen in Table 1, the convergence accuracy obtained by using the BCLS dwell time algorithm was found 
Table 1 Comparison of simulation results for different dwell time algorithms

\begin{tabular}{|c|c|c|c|c|c|}
\hline \multirow[t]{2}{*}{ Index } & \multirow{2}{*}{$\begin{array}{l}\text { Main simulation parameters } \\
\text { Acceleration (g) }\end{array}$} & \multicolumn{2}{|c|}{$\begin{array}{l}\text { BCLS-based dwell time algorithm Simulation } \\
\text { results }\end{array}$} & \multicolumn{2}{|c|}{$\begin{array}{l}\text { NNLS-based dwell time algorithm Simu- } \\
\text { lation results }\end{array}$} \\
\hline & & Surface error PV $(\lambda)$ & $\begin{array}{l}\text { Surface error } \\
\text { RMS }(\lambda)\end{array}$ & Surface error PV $(\lambda)$ & $\begin{array}{l}\text { Surface } \\
\text { error RMS } \\
(\lambda)\end{array}$ \\
\hline 1 & 0.05 & 0.029571 & 0.003906 & 0.106895 & 0.026035 \\
\hline 2 & 0.1 & 0.026082 & 0.002746 & 0.044123 & 0.006126 \\
\hline 3 & 0.2 & 0.026051 & 0.002058 & 0.039592 & 0.002596 \\
\hline 4 & 0.4 & 0.023020 & 0.002008 & 0.024158 & 0.002083 \\
\hline 5 & 0.8 & 0.022324 & 0.001980 & 0.025399 & 0.002010 \\
\hline
\end{tabular}

(a)

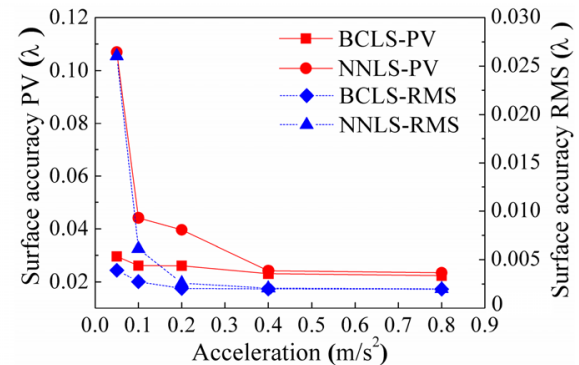

(b)

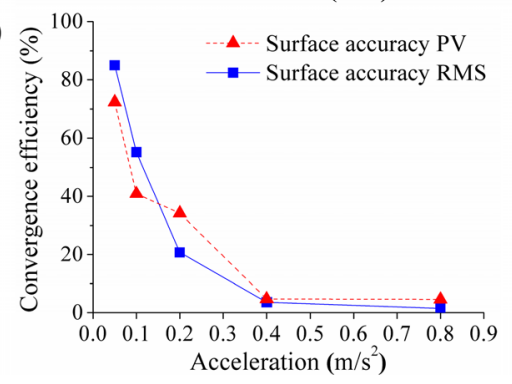

Fig. 12 Comparison of dwell time simulations by using BCLS and NNLS algorithms. a Surface accuracy; (b) convergence accuracy

to be higher than that of the NNLS algorithm. This is the situation for both surface error models. When accelerating to a value $a \geq 0.4 \mathrm{~g}$, the surface figure accuracy PV and RMS of BCLS-algorithm was found to be $3.60 \%$ and $4.71 \%$ lower than that of the NNLS algorithm, respectively. This indicates that the convergence accuracy obtained by the BCLS algorithm is slightly higher than the accuracy obtained by the NNLS algorithm.

Figure 12 shows the proportion of the convergence accuracy improvement of the BCLS algorithm, in relation to the NNLS algorithm, in improving the accuracy of the surface error. The smaller the acceleration, the higher the convergence efficiency of the BCLS-algorithm. When $a$ is down to $0.1 \mathrm{~g}$, the surface accuracies (of types PV and RMS) increase by as much as $40.8 \%$ and $55.2 \%$, respectively, when using the BCLS algorithm, which is not the case with the NNLS algorithm. This result demonstrates that the convergence accuracy of the BCLS algorithm is apparently better than that of the NNLS algorithm, especially when the acceleration is exceptionally low.

The reason that the convergence accuracy of the BCLSbased algorithm is higher than that of the NNLS-algorithm, is that the former algorithm takes into account the influence of acceleration and deceleration of the movable axis in calculating the dwell time, while the latter do not. There is just a small difference between the dwell time calculated by the BCLS-based algorithm and the engineering practice. On the contrary, the dwell time calculated by the NNLS-algorithm is quite different. The reason is that the dwell time needs extra correction in the post-processing to meet the actual demand, and this will reduce the convergence accuracy.

\subsubsection{Influence of Acceleration on the Convergence Accuracy of Surface Error}

The acceleration of the machine axis has been set to $0.8 \mathrm{~g}, 0.4 \mathrm{~g}, 0.2 \mathrm{~g}, 0.1 \mathrm{~g}, 0.05 \mathrm{~g}, 0.025 \mathrm{~g}$, and $0.0125 \mathrm{~g}$. In addition, the speed has been set to be within the range $200 \sim 4000 \mathrm{~mm} / \mathrm{min}$. The influence of acceleration on the total dwell time, the calculated maximum speed, and the surface figure accuracy (of types PV and RMS) are shown in Fig. 13. As can be seen in Fig. 13a, the lower the acceleration, the longer the total dwell time. When the acceleration is larger than $0.1 \mathrm{~g}\left(\approx 0.98 \mathrm{~m} / \mathrm{s}^{2}\right)$, the total dwell time will just slightly change. However, when the acceleration is less than $0.1 \mathrm{~g}$, the total dwell time increases sharply with a decrease in acceleration. As shown in Fig. 13b, the lower the acceleration, the lower the calculated maximum speed. Moreover, when the acceleration is larger than $0.2 \mathrm{~g}$ $\left(\approx 1.96 \mathrm{~m} / \mathrm{s}^{2}\right.$ ), the calculated maximum speed stays at the restricted upper speed. Also, when the acceleration is less than $1.96 \mathrm{~m} / \mathrm{s}^{2}$, the calculated speed depends on the acceleration. It is close to the characteristic speed, which is consistent with Eq. (19). This indicates that the convergence accuracy of the surface error can only be guaranteed when the speed of the axis falls within the restricted range, even 
(a)

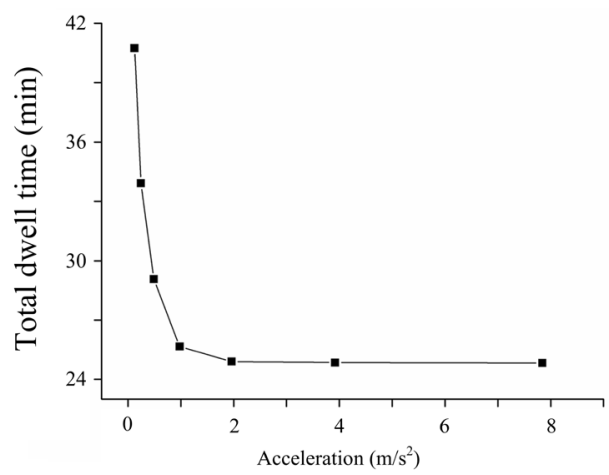

(b)

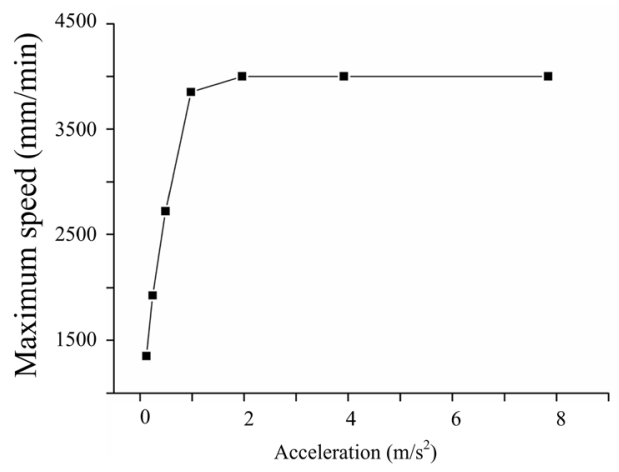

(c)

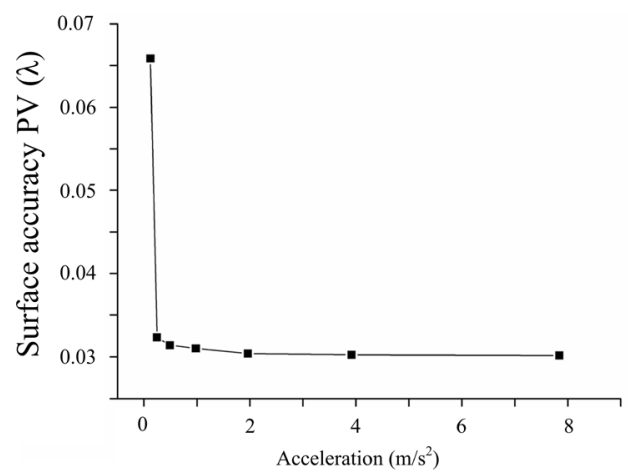

(d)

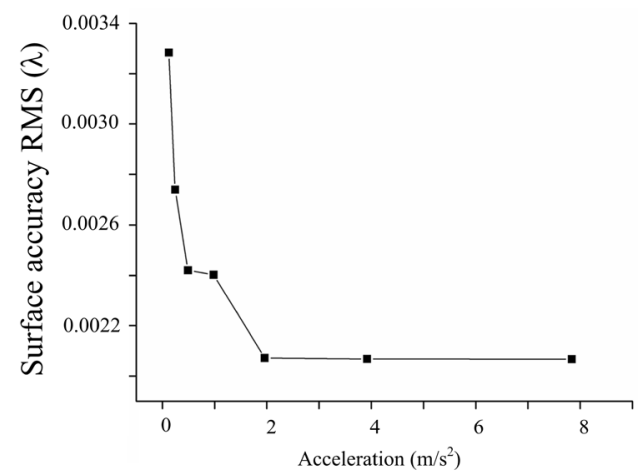

Fig. 13 Influence of the acceleration on the (a) total dwell time, (b) calculated maximum speed, (c) surface accuracy (of type PV), and (d) surface accuracy (of type RMS) if the acceleration performance is poor. Figure $13 \mathrm{c}, \mathrm{d}$ demonstrate that the machine axis acceleration has little effect on the convergence accuracy of the surface error (of type $\mathrm{PV}$ and RMS). This indicates that a lower acceleration performance can still achieve a high convergence accuracy.

The simulation results showed that the reduction in machine tool acceleration has a little effect on the machining accuracy but brings a large influence on the total polishing time. When the acceleration decreases to $0.1 \mathrm{~g}$ (which can be easily realized by using ordinary CNC machine tools), the polishing machine possesses a remarkably high machining accuracy and efficiency. Meanwhile, a too high machine tool accuracy has little effect on the machining accuracy and efficiency. On the contrary, it would largely increase the cost of the machine tools. Therefore, when using the proposed algorithm, a moderate machine tool performance will ensure a good machine tool convergence accuracy and machining efficiency.

\subsubsection{Influence of Machine Axis Speed on the Convergence Accuracy of the Surface Error}

The machine axis acceleration was set to $0.3 \mathrm{~g}$ and, thus, the calculated characteristic speed $v^{*}$ was $6675.6 \mathrm{~mm} /$ min. Moreover, the maximum speeds were set to $1.2 v^{*}$, $1.0 v^{*}, 0.8 v^{*}, 0.6 v^{*}, 0.4 v^{*}, 0.3 v^{*}$, and $0.2 v^{*}$. The modulation ratio of maximum to minimum speed was 20.0. The influence of the axis speed on the maximum speed, total dwell time, and surface figure accuracy (of types PV and RMS), are shown in Fig. 14. As can be seen in Fig. 14b, the total dwell time increases with a decrease in axis speed. When the set speed is not higher than the characteristic speed, the calculated maximum speed is close to the characteristic speed (as shown in Fig. 14a). Furthermore, when the set speed is reduced to $0.7 v^{*}$, the surface errors (of types PV and RMS) show minima values, and the surface errors would slightly increase if the speed is further reduced (which is shown in Fig. 14c, d). While the set speed exceeds the characteristic speed, the maximum speed would not exceed the characteristic speed (see Fig. 14a). Also, when the set speed is too small, it would result in a deterioration of surface figure accuracy and a reduction in machining efficiency.

The results from the speed simulations demonstrate that a too high axis speed is meaningless due to the limitation of the characteristic speed. Also, a too low speed will result in a longer polishing time and lower convergence efficiency. Therefore, the set speed is generally advised to be set as 0.6-0.8 times of the characteristic speed, which ensures high convergence accuracy and a relatively shorter polishing time. 
(a)

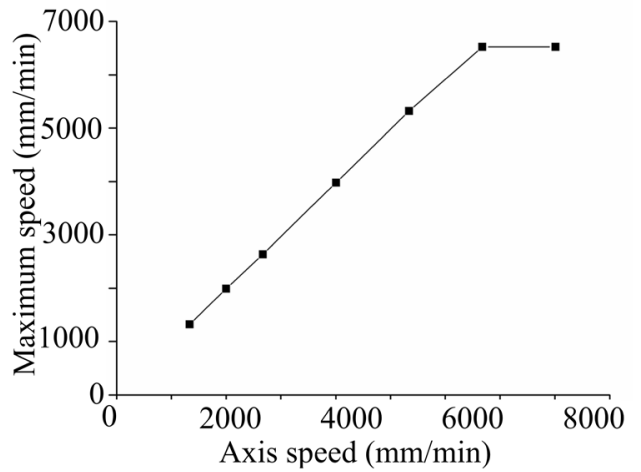

(b)

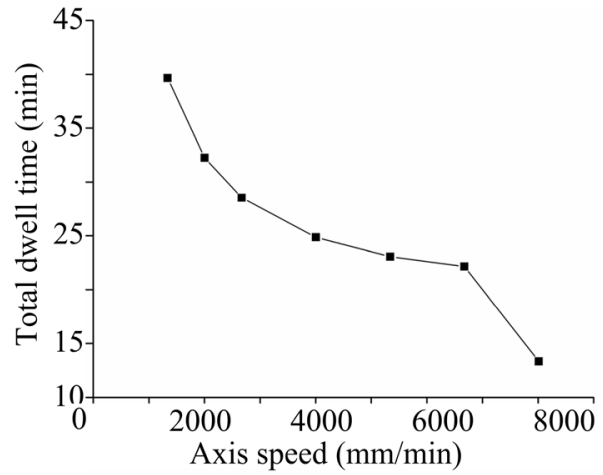

(c)

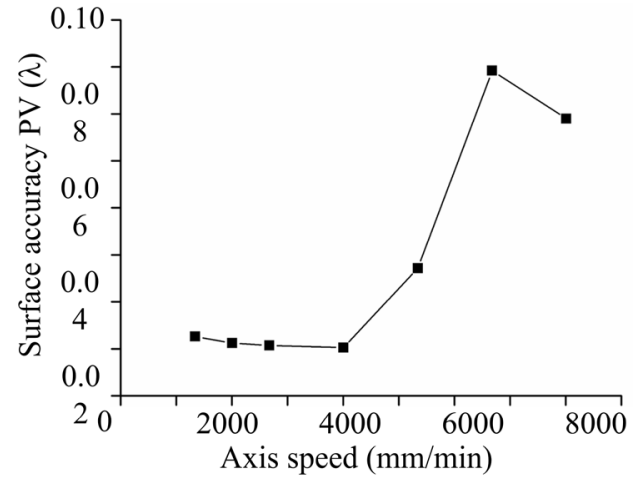

(d)

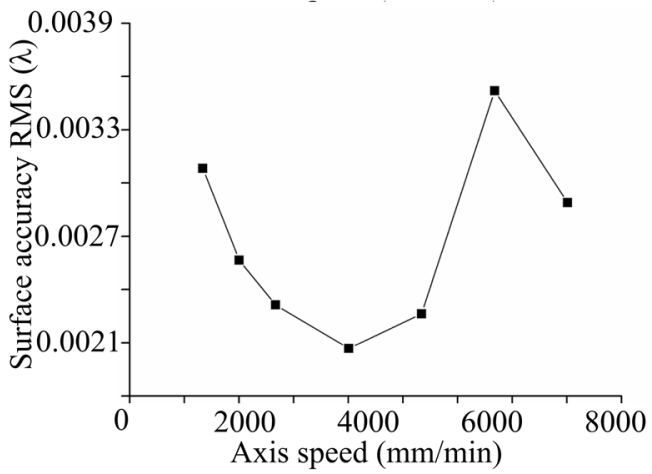

Fig. 14 Influence of speed on (a) calculated maximum speed, (b) total dwell time, (c) surface figure accuracy PV, (d) surface figure accuracy RMS

\section{Conclusions}

To improve the convergence accuracy of the surface error, a novel BCLS-based dwell time algorithm, under dynamic performance constraints of the machine tool, has been developed in the present work. Computational methods have dealt with the boundary constraints, which are all included in the algorithm that handles the numerical calculations of the dwell time model. The high precision convergence efficiency of the BCLS-algorithm has been validated by using both simulations and experiments. Moreover, the influence of different machine tool dynamic performances on the dwell time solution has also been discussed. The main conclusions are as follows:

(1) The proposed BCLS-algorithm shows remarkably high convergence accuracy, in addition to a strong adaptability to machine tools with different dynamic performances. Also, the dwell time solution automatically satisfies the special requirements of different machine tools. For the polishing machine tool with excellent machine tool performances, the convergence accuracy of the BCLS-algorithm may be slightly higher than that of the common dwell time algorithm. However, for polishing machine tools whose machine performance is not excellent enough, the BCLS-algorithm also ensures high convergence accuracy.

(2) The machine tool acceleration was shown to have little effect on the machining accuracy, but it greatly affects the total polishing time. Moderate machine tool accuracy will ensure high machine tool convergence accuracy and machining efficiency.

(3) When the machine axis speed is too small, it leads to a deterioration of the surface figure accuracy and a decrease in machining efficiency. The maximum speed is generally advised to be set to $0.6-0.8$ times of the characteristic speed, to ensure a high convergence accuracy and a relatively short polishing time.

The research on the dwell time algorithm under dynamic performance constraints of machine tool, not only ensures the machining accuracy, but also reduces the machine tool accuracy and saves resources, which is of great significance for the development of green technology in the field of ultra-precision machining.

Author Contributions All authors have contributed to the study conception and design. YZ conceived and designed the experiments, performed the experiments and analyzed the data. WF gave some advices on formula derivation. FF and WH supervised the whole project and gave advices on manuscript editing. YZ wrote the paper. All authors read and approved the final manuscript. 
Funding The Science Challenge Project (No.TZ2016006-0504-01); the National Natural Science Foundation of China (No. 52005463); High-end CNC Machine Tools and Basic Manufacturing Equipment Major National Science and Technology Project (2017ZX04022001);, Laboratory of Precision Manufacturing Technology, CAEP.

Data Availability Not applicable.

Code Availability Not applicable.

\section{Compliance with Ethical Standards}

Conflict of Interest The authors declare no conflicts of interest. The funders had no role in the design of the study; in the collection, analyses, or interpretation of data; in the writing of the manuscript, or in the decision to publish the results.

Open Access This article is licensed under a Creative Commons Attribution 4.0 International License, which permits use, sharing, adaptation, distribution and reproduction in any medium or format, as long as you give appropriate credit to the original author(s) and the source, provide a link to the Creative Commons licence, and indicate if changes were made. The images or other third party material in this article are included in the article's Creative Commons licence, unless indicated otherwise in a credit line to the material. If material is not included in the article's Creative Commons licence and your intended use is not permitted by statutory regulation or exceeds the permitted use, you will need to obtain permission directly from the copyright holder. To view a copy of this licence, visit http://creativecommons.org/licenses/by/4.0/.

\section{References}

1. Hu, H., Qi, E., Luo, X., Zhang, X., \& Xue, D. (2018). Rapid fabrication strategy for $\mathrm{O} .15 \mathrm{~m}$ off-axis parabolic parts using computer-controlled optical surfacing. Applied Optics, 57(34), F37-F43.

2. Suratwala, T. I., Steele, W. A., Wong, L. A., \& Tham, G. C. (2019). Subsurface mechanical damage correlations after grinding of various optical materials. Optical Engineering, 58(09), 092604.

3. Sidpara, A. (2014). Magnetorheological finishing: a perfect solution to nanofinishing requirements. Optical Engineering, 53(9), 092002.

4. Lu, Y., Xie, X., Zhou, L., Dai, Z., \& Chen, G. (2017). Improve optics fabrication efficiency by using a radio frequency ion beam figuring tool. Applied Optics, 56(2), 260-266.

5. Yin, X., Deng, W., Tang, W., Zhang, B., Xue, D., Zhang, F., et al. (2016). Ion beam figuring approach for thermally sensitive space optics. Applied Optics, 55(28), 8049-8055.

6. Zhong, B., Wang, C., Chen, X., \& Wang, J. (2019). Time-varying tool influence function model of bonnet polishing for aspheric surfaces. Applied Optics, 58(4), 1101-1109.

7. Zhong, B., Chen, X., Pan, R. P., Jian, W., et al. (2017). The effect of tool wear on the removal characteristics in high-efficiency bonnet polishing. International Journal of Advanced Manufacturing Technology, 91(9-12), 3653-3662.

8. Nguyen, T., \& Wang, J. (2019). A review on the erosion mechanisms in abrasive waterjet micromachining of brittle materials. International Journal of Extreme Manufacturing, 1(1), 012006.

9. Fang, F., Zhang, N., Guo, D., Ehmann, K., Cheung, B., Liu, K., et al. (2019). Towards atomic and close-to-atomic scale manufacturing. International. Journal of Extreme Manufacturing, 1(1), 012001 .

10. Guo, Z., Jin, T., Xie, G., Lu, A., \& Qu, M. (2019). Approaches enhancing the process accuracy of fluid jet polishing for making ultra-precision optical components. Precision Engineering, 56, 20-37.

11. Natarajan, Y., Murugasen, P. K., Sundarajan, L. R., et al. (2019). Experimental investigation on cryogenic assisted abrasive water jet machining of aluminium alloy. International Journal of Precision Engineering and Manufacturing-Green Technology., 6, 415-432.

12. Kum, C. W., Sato, T., Guo, J., Liu, K., \& Butler, D. (2018). A novel media properties-based material removal rate model for magnetic field-assisted finishing. International Journal of Mechanical Sciences, 141, 189-197.

13. Schneider, F., Das, J., Kirsch, B., et al. (2019). Sustainability in ultra precision and micro machining: a review. International Journal of Precision Engineering and Manufacturing-Green Technology., 6, 601-610.

14. Li, L., Liu, Z., Xue, D., Deng, W., Li, R., Bai, Y., et al. (2018). Rapid fabrication of a lightweight $2 \mathrm{~m}$ reaction-bonded $\mathrm{SiC}$ aspherical mirror. Results in Physics, 10, 903-912.

15. McNeil, S. R. W. J. R. (1987). Neutral ion beam figuring of large optical surfaces. Proc SPIE, 818, 320-324.

16. Xu C, Aissaoui I, Jacquey S (1994) Algebraic analysis of the Van Cittert iterative method of deconvolution with a general relaxation factor. Journal of the Optical Society of America A 11(11).

17. Dong, Z., Cheng, H., \& Tam, H. Y. (2014). Modified dwell time optimization model and its applications in subaperture polishing. Applied Optics, 53(15), 3213-3224.

18. Jiao, C., Li, S., \& Xie, X. (2009). Algorithm for ion beam figuring of low-gradient mirrors. Applied Optics, 48(21), 4090-4096.

19. Carnal, C. L., Egert, C. M., \& Hylton, K. W. (1992). Advanced matrix-based algorithm for ion-beam milling of optical components. SPIE, 1752, 54-62.

20. Cao, Z. C., Cheung, C. F., \& Liu, M. Y. (2018). Model-based self-optimization method for form correction in the computer controlled bonnet polishing of optical freeform surfaces. Optics Express, 26(2), 2065-2078.

21. Jiao, C. (2009). Bayesian principle based dwell time algorithm for ion beam figuring of low gradient mirrors. Journal of Mechanical Engineering, 45(11), 253.

22. Zhu W, Beaucamp A (2019) Zernike mapping of optimum dwell time in deterministic fabrication of freeform optics. Optics Express, 27(20).

23. Wu, J. F., Lu, Z. W., Zhang, H. X., \& Wang, T. S. (2009). Dwell time algorithm in ion beam figuring. Applied Optics, 48(20), 3930-3937.

24. Yang HLM (2001) Dwell time algorithm for computer-controlled polishing of small axis-symmetrical aspherical lens mold. Optical Engineering, 40(9).

25. Deng, W. (2007). Dwell time algorithm based on matrix algebra and regularization method. Optics and Precision Engineering, 15(7), 1009-1015.

26. Dong, Z., Cheng, H., \& Tam, H. Y. (2015). Robust linear equation dwell time model compatible with large scale discrete surface error matrix. Applied Optics, 54(10), 2747-2756.

27. Zhou, L., Dai, Y., Xie, X., \& Li, S. (2010). Optimum removal in ion-beam figuring. Precision Engineering, 34(3), 474-479.

28. Longxiang, L., Weijie, D., Binzhi, Z., Yang, B., Ligong, Z., \& Xuejun, Z. (2014). Dwell time algorithm for large aperture optical element in magnetorheological finishing. Acta Optica Sinica, 34(5), 217-223.

29. Dong, Z., \& Cheng, H. (2015). Toward the complete practicability for the linear-equation dwell time model in subaperture polishing. Applied Optics, 54(30), 8884-8890. 
30. Song, C., Dai, Y., \& Peng, X. (2010). Model and algorithm based on accurate realization of dwell time in magnetorheological finishing. Applied Optics, 49(19), 3676-3683.

31. Reci, A., Sederman, A. J., \& Gladden, L. F. (2017). Obtaining sparse distributions in 2D inverse problems. Journal of Magnetic Resonance, 281, 188-198.

32. Li, L., Zheng, L., Deng, W., Wang, X., Wang, X., Zhang, B., et al. (2015). Optimized dwell time algorithm in magnetorheological finishing. International Journal of Advanced Manufacturing Technology, 81(5-8), 833-841.

33. Park, C., Kim, H., Cho, H., et al. (2019). Effect of relative surface charge of colloidal silica and sapphire on removal rate in chemical mechanical polishing. International Journal of Precision Engineering and Manufacturing-Green Technology., 6, 339-347.

34. Zhou, Z., Yuan, X., Gu, M., Qiu, M., zhou, 1., \& li, y. (2017). Solution algorithm of dwell time in slope-based figuring model. Paper presented at the AOPC 2017: Optoelectronics and Micro/ Nano-Optics.

35. Song, C., Dai, Y.-F., \& Peng, X.-Q. (2013). Magnetorheological finishing of low-gradient curved surfaces based on four-axis linkage technique. Journal of Central South University, 20(9), 2349-2358.

36. Piccolomini, E. L., \& Zama, F. (2011). An iterative algorithm for large size least-squares constrained regularization problems. Applied Mathematics and Computtation, 217(24), 10343-10354.

Publisher's Note Springer Nature remains neutral with regard to jurisdictional claims in published maps and institutional affiliations.

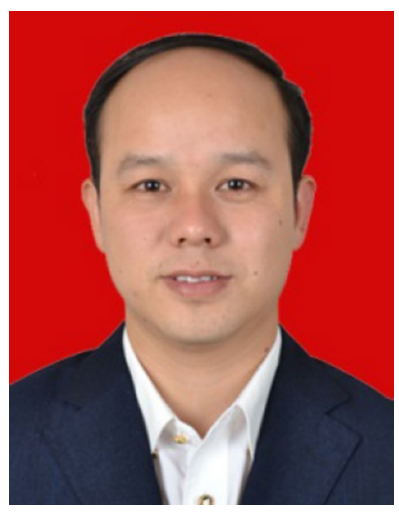

Yunfei Zhang received the B.Sc. degree from the School of Mechanical Science and Engineering, Huazhong University of Science and Technology, Wuhan, China in 2007 and M.Sc. degree from China Academy of Engineering Physics (CAEP), Mianyang, China in 2010. He is currently an Associate Professor at CAEP. His research interests mainly include magnetorheological finishing (MRF), theory and technology of computercontrolled optical manufacturing.

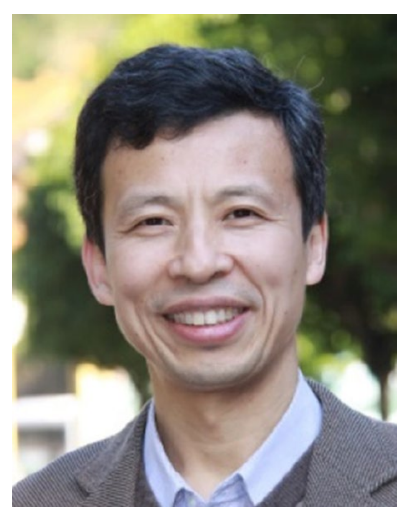

Fengzhou Fang is a joint Professor and the director of Centre of Micro/Nano Manufacturing Technology (MNMT) at Tianjin University and University College Dublin. He has been doing both fundamental studies and application development in the areas of freeform optics design and manufacturing, micro/nano manufacturing, ACSM, ultraprecision machining and measurement benefiting a variety of industries in medical devices, bio-implants, optics and molds sectors.

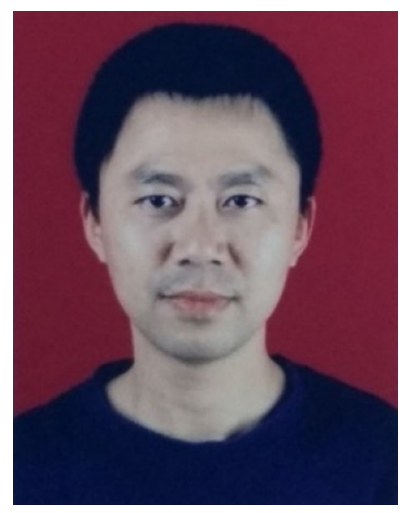

Wen Huang received the B.Sc. degree from the University of Science and Technology Beijing, Beijing, China in 1997 and M.Sc. degree from University of Electronic Science and Technology, Chengdu, China in 2002. $\mathrm{He}$ is currently a Professor at China Academy of Engineering Physics (CAEP). His research interests include various fields of precision engineering, advanced optics manufacturing and so on.

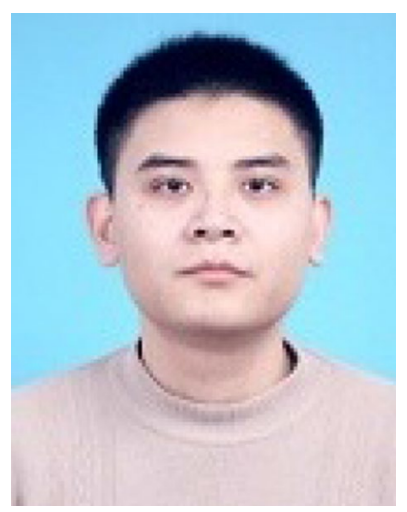

Wei Fan received the B.S. degree in mathematics and applied mathematics from Huazhong University of Science and Technology, Wuhan, China, in 2007, and the Ph.D. degrees in applied mathematics from Academy of Mathematics and Systems Science, Chinese Academy of Sciences, Beijing, China, in 2012. $\mathrm{He}$ is currently an Associate Professor, and Master's Supervisor with the Institute of Mechanical Manufacturing Technology, China Academy of Engineering Physics. He is a Senior Member of Chinese Mechanical Engineering Society (CMES). He has authored or coauthored more than 30 international journal papers and conference papers in the fields of $\mathrm{CAD} / \mathrm{CAM} / \mathrm{CNC}$ technology, advanced optics manufacturing. 